\title{
Theoretical Study of Positronic Atoms with Adiabatic Separation of Positronic Motion
}

\author{
K. Strasburger and M. Wolcyrz
}

Institute of Physical and Theoretical Chemistry

Wrocław University of Technology

Wybrzeże Wyspiańskiego 27, 50-370 Wrocław, Poland

\begin{abstract}
A modified adiabatic approximation, with the positron treated as a light nucleus and its charge included only partially in the electronic Hamiltonian, was applied to compute the energies and properties of bound states of lithium positride, positronic beryllium and positronic magnesium. The electronic Schrödinger equation was solved with the configuration interaction method. No bound state was obtained for lithium positride, while the dissociation energies of positronic beryllium and positronic magnesium were underestimated by 2.6 and 7 millihartrees, respectively. Consequences for the applicability of the adiabatic approximation to describe positronic systems are discussed.
\end{abstract}

PACS numbers: 34.20.Cf, 31.50.Bc, 36.10.Dr

\section{Introduction}

Ten years ago a breakthrough took place in theoretical studies of positronic atoms. Stability of positronic lithium - the first known case of positron binding by a neutral atom - has been demonstrated in two independent but quite similar works $[1,2]$, with the same type of trial wave functions, namely explicitly correlated Gaussian (ECG) functions, differing only in the strategies of optimization of nonlinear parameters (stochastic versus deterministic) and sizes of basis sets. Further calculations on various positronic atoms quickly followed (see [3] for a review and [4-8] for more recent results). Besides of variational calculations with ECG functions, diffusion quantum Monte Carlo simulations appeared to be able to provide accurate energies for positronic systems [9-13]. Unfortunately, the computational cost of both methods increases quickly with the number of active electrons, limiting in practice their applicability to rather small atoms and molecules.

The configuration interaction (CI) method, which is well established in computational quantum chemistry, can be adapted to handle also positronic matter. However, this method faces a different problem. The convergence of the expansion 
of the wave function in terms of configurations built from one-particle orbitals is extremely slow, especially for the annihilation rate [14]. This means that orbitals of very high angular momentum should be included in the basis set to provide reasonable results. Of course, calculation of necessary integrals with such orbital basis set becomes very expensive. Appropriate techniques, connected with extrapolation to infinite basis set, have been developed and applied to atomic systems only [15-20]. CI calculations for positronic molecules, carried out up to date, provided at best some qualitative information [21-25].

The positron was treated on equal footing with electrons in all the above mentioned methods. Fully different philosophy of treating the positron as a light pseudonucleus was used in the works of Stachowiak and Boroński [26] and Mohallem et al. [27-30]. The idea of separation of the positronic motion from the electronic one appeared even earlier - the nonexistence of some bound positronic atoms has been shown within such approach [31]. The drawback of such separation is that it bears an irremovable, intrinsic error to all properties of the studied system. On the other hand, it introduces the familiar notion of potential energy surface to the positron chemistry and makes it possible to use the whole mature technology of computational quantum chemistry to obtain this potential energy. In our previous paper [32], we formulated the adiabatic method in such a manner that the leptonic energy is variationally bounded. We presented also some preliminary results, but finding the limits of applicability and estimation of the intrinsic error of our method requires more comparisons with accurate energies and annihilation rates of various positronic systems. The gathered data on positronic atoms is a good reference.

\section{Method}

Details of the computational method used by us were discussed in our previous article [32]. Therefore, this method will be only shortly sketched here. The problem to be solved is the leptonic Schrödinger equation for fixed nuclear positions. Let us assume that only one positron is present, and its coordinates will be denoted as $\boldsymbol{r}_{\mathrm{p}}$, while $\boldsymbol{r}_{\mathrm{e}}$ will denote coordinates of all electrons in an atom or molecule. Adiabatic separation of electronic and positronic motions is postulated by the product form of trial wave function,

$$
\Psi_{\text {lep }}\left(\boldsymbol{r}_{\mathrm{p}}, \boldsymbol{r}_{\mathrm{e}}\right)=\Psi_{\mathrm{p}}\left(\boldsymbol{r}_{\mathrm{p}}\right) \Psi_{\mathrm{e}}\left(\boldsymbol{r}_{\mathrm{p}}, \boldsymbol{r}_{\mathrm{e}}\right) .
$$

The leptonic Hamiltonian gets partitioned $\left(\hat{H}_{\text {lep }}=\hat{H}_{\mathrm{e}}+\hat{H}_{\mathrm{p}}\right)$ and the electronic wave function $\left(\Psi_{\mathrm{e}}\right)$ has to fulfill the electronic Schrödinger equation

$$
\hat{H}_{\mathrm{e}} \Psi_{\mathrm{e}}\left(\boldsymbol{r}_{\mathrm{p}}, \boldsymbol{r}_{\mathrm{e}}\right)=E_{\mathrm{e}}\left(\boldsymbol{r}_{\mathrm{p}}\right) \Psi_{\mathrm{e}}\left(\boldsymbol{r}_{\mathrm{p}}, \boldsymbol{r}_{\mathrm{e}}\right)
$$

for any fixed positronic coordinates. Conceptually, this step is the same as for the separation of motions of true heavy nuclei from those of light particles. However, in order to simulate the finite (and small) positron mass effect on the distribution of the electron density around this particle, the positronic charge is included only partially into the electronic Hamiltonian 


$$
\hat{H}_{\mathrm{e}}=-\frac{1}{2} \sum_{i=1}^{N_{\mathrm{e}}} \nabla_{i}^{2}-\sum_{A=1}^{N_{\text {nuc }}} \sum_{i=1}^{N_{\mathrm{e}}} \frac{Z_{A}}{r_{A i}}+\sum_{i>j}^{N_{\mathrm{e}}} \frac{1}{r_{i j}}+\sum_{A=1}^{N_{\text {nuc }}} \frac{Z_{A} q_{\mathrm{p}}}{r_{A \mathrm{p}}}-\sum_{i=1}^{N_{\mathrm{e}}} \frac{q_{\mathrm{p}}}{r_{i \mathrm{p}}} .
$$

The quantity $q_{\mathrm{p}}$ may be treated as a variational parameter. Remaining part of positronic charge appears in the positronic Hamiltonian

$$
\hat{H}_{\mathrm{p}}=-\frac{1}{2} \nabla_{\mathrm{p}}^{2}+\sum_{A=1}^{N_{\text {nuc }}} \frac{Z_{A}\left(1-q_{\mathrm{p}}\right)}{r_{A \mathrm{p}}}-\sum_{i=1}^{N_{\mathrm{e}}} \frac{1-q_{\mathrm{p}}}{r_{i \mathrm{p}}}
$$

and the equation for positronic wave function

$$
\left[-\frac{1}{2} \nabla_{\mathrm{p}}^{2}+E_{\mathrm{e}}\left(\boldsymbol{r}_{\mathrm{p}}\right)+V_{\mathrm{es}}\left(\boldsymbol{r}_{\mathrm{p}}\right)+E_{\mathrm{ad}}\left(\boldsymbol{r}_{\mathrm{p}}\right)\right] \Psi_{\mathrm{p}}\left(\boldsymbol{r}_{\mathrm{p}}\right)=E_{\mathrm{lep}} \Psi_{\mathrm{p}}\left(\boldsymbol{r}_{\mathrm{p}}\right)
$$

contains, besides the adiabatic correction

$$
E_{\mathrm{ad}}=\left\langle\Psi_{\mathrm{e}}\left(\boldsymbol{r}_{\mathrm{p}}, \boldsymbol{r}_{\mathrm{e}}\right)\left|-\frac{1}{2} \nabla_{\mathrm{p}}^{2}\right| \Psi_{\mathrm{e}}\left(\boldsymbol{r}_{\mathrm{p}}, \boldsymbol{r}_{\mathrm{e}}\right)\right\rangle,
$$

also the electrostatic correction

$$
V_{\mathrm{eS}}\left(\boldsymbol{r}_{\mathrm{p}}\right)=\sum_{A=1}^{N_{\text {nuc }}} \frac{Z_{A}\left(1-q_{\mathrm{p}}\right)}{r_{A \mathrm{p}}}-\left\langle\Psi_{\mathrm{e}}\left(\boldsymbol{r}_{\mathrm{p}}, \boldsymbol{r}_{\mathrm{e}}\right)\left|\sum_{i=1}^{N_{\mathrm{e}}} \frac{1-q_{\mathrm{p}}}{r_{i \mathrm{p}}}\right| \Psi_{\mathrm{e}}\left(\boldsymbol{r}_{\mathrm{p}}, \boldsymbol{r}_{\mathrm{e}}\right)\right\rangle .
$$

Therefore, the effective positronic charge $q_{\mathrm{p}}$, occurring in the electronic equation, is only a partitioning parameter which determines the form of the electronic wave function, the potential for positronic motion and, consequently, also the positronic wave function and the leptonic energy. Optimal charge $q_{\mathrm{p}}$ gives the lowest value of $E_{\text {lep. }}$.

The potential in Eq. (5) is usually shifted, so that it goes to zero at infinite positron-nucleus distance

$$
\begin{aligned}
& V_{\mathrm{p}}\left(\boldsymbol{r}_{\mathrm{p}}\right)=E_{\mathrm{e}}\left(\boldsymbol{r}_{\mathrm{p}}\right)+V_{\mathrm{es}}\left(\boldsymbol{r}_{\mathrm{p}}\right)+E_{\mathrm{ad}}\left(\boldsymbol{r}_{\mathrm{p}}\right)-E_{\mathrm{e}}\left(r_{\mathrm{p}} \rightarrow \infty\right) \\
& \quad-V_{\mathrm{es}}\left(r_{\mathrm{p}} \rightarrow \infty\right)-E_{\mathrm{ad}}\left(r_{\mathrm{p}} \rightarrow \infty\right) .
\end{aligned}
$$

This operation does not change $\Psi_{\mathrm{p}}$, but the signs of eigenvalues (positronic states energies) of the following equation, containing the shifted potential

$$
\left[\hat{T}_{\mathrm{p}}+V_{\mathrm{p}}\left(\boldsymbol{r}_{\mathrm{p}}\right)\right] \Psi_{\mathrm{p}}\left(\boldsymbol{r}_{\mathrm{p}}\right)=E_{\mathrm{p}} \Psi_{\mathrm{p}}\left(\boldsymbol{r}_{\mathrm{p}}\right)
$$

carry the basic information about the character of respective eigenfunctions. $E_{\mathrm{p}}<0$ indicates that a bound state has been obtained. This is the necessary condition to establish binding, but it is not sufficient, if a given positronic system dissociates to the positronium atom. The dissociation energy of an AB system (let A be an atom or molecule)

$$
D_{0}(\mathrm{AB})=E_{\mathrm{lep}}(\mathrm{A})+E_{\mathrm{lep}}(\mathrm{B})-E_{\mathrm{lep}}(\mathrm{AB})
$$

is always equal to $-E_{\mathrm{p}}$ for $\mathrm{B}=\mathrm{e}^{+}$. However, for $\mathrm{B}=\mathrm{Ps}$, the optimal value of $q_{\mathrm{p}}(\mathrm{B})$ is 0.5 [32], but optimal $q_{\mathrm{p}}(\mathrm{AB})$ maybe different. In such situation, establishing the energetic stability of $\mathrm{AB}$ requires explicit calculation of $D_{0}$ with Eq. (10).

Besides of the energy, the most interesting properties of positronic systems are those related to annihilation. Electron density on the positron, readily available 
from the leptonic function

$$
d_{\mathrm{e}}\left(\boldsymbol{r}_{\mathrm{p}}\right)=\left\langle\Psi_{\mathrm{e}}\left(\boldsymbol{r}_{\mathrm{p}}, \boldsymbol{r}_{\mathrm{e}}\right)\left|\sum_{i=1}^{N_{\mathrm{e}}} \delta\left(\boldsymbol{r}_{i}-\boldsymbol{r}_{\mathrm{p}}\right)\right| \Psi_{\mathrm{e}}\left(\boldsymbol{r}_{\mathrm{p}}, \boldsymbol{r}_{\mathrm{e}}\right)\right\rangle,
$$

multiplied by the positron density for given $\boldsymbol{r}_{\mathrm{p}}=\boldsymbol{R}$ :

$$
d_{\mathrm{p}}(\boldsymbol{R})=\left\langle\Psi_{\text {lep }}\left|\delta\left(\boldsymbol{r}_{\mathrm{p}}-\boldsymbol{R}\right)\right| \Psi_{\text {lep }}\right\rangle=\left|\Psi_{\mathrm{p}}(\boldsymbol{R})\right|^{2}
$$

yields the electron-positron contact density

$$
d_{\mathrm{ep}}(\boldsymbol{R})=\left\langle\Psi_{\text {lep }}\left|\sum_{i=1}^{N_{\mathrm{e}}} \delta\left(\boldsymbol{r}_{i}-\boldsymbol{R}\right) \delta\left(\boldsymbol{r}_{\mathrm{p}}-\boldsymbol{R}\right)\right| \Psi_{\mathrm{lep}}\right\rangle=d_{\mathrm{e}}(\boldsymbol{R}) \mathrm{d}_{\mathrm{p}}(\boldsymbol{R}) .
$$

Integrating $d_{\mathrm{ep}}$ over $\boldsymbol{R}$ leads to the electron-positron coalescence

$$
\left\langle\delta_{\mathrm{ep}}\right\rangle=\left\langle\Psi_{\text {lep }}\left|\sum_{i=1}^{N} \delta\left(\boldsymbol{r}_{i}-\boldsymbol{r}_{\mathrm{p}}\right)\right| \Psi_{\text {lep }}\right\rangle=\int_{V} d_{\mathrm{ep}}(\boldsymbol{R}) \mathrm{d} \boldsymbol{R},
$$

to which the two-photon annihilation rate is directly proportional. For closed-shell electronic subsystems and $\left\langle\delta_{\text {ep }}\right\rangle$ expressed in atomic units, respective formula can be written as

$$
\Gamma_{2}=\pi \alpha^{4} a_{0}^{-1} c\left\langle\delta_{\mathrm{ep}}\right\rangle .
$$

\section{Details of calculations}

In our previous work [32], in which the method was tested for model of one-electron atoms with fractional nuclear charges and for positronium hydride, the electronic Schrödinger equation was solved in the basis of floating spherical (for one-electron systems) or explictly correlated Gaussian functions (for PsH). Parameters of these functions - exponents and positions of centers - were individually optimized for each positron-nucleus distance, giving very accurate potential energy curves for positronic motion. We estimated that their errors did not exceed a few microhartrees. Discrepancies of those calculations with almost exact reference results were to be attributed to the intrinsic error of the adiabatic approximation. Such an approach would be impossible to be realized in the present work, because of larger number of electrons in the studied atoms. Computing even a single point of the potential energy curve for a 4-electron system in the basis of ECG functions, with accuracy comparable to that achieved in the previous work, would be a very demanding and time consuming task. Therefore, we used the configuration interaction method with frozen electronic cores.

Our calculations were carried out in a fixed, finite orbital basis set, which was the source of additional errors, unrelated to the adiabatic approximation. The first one was the basis set superposition error, which was corrected within the counterpoise procedure - in computation of the potential defined by Eq. (8), the electronic energy $E_{\mathrm{e}}$ and electrostatic correction $V_{\mathrm{es}}$ (the latter only for atoms dissociating to positronium) of isolated species were obtained in supermolecular basis set. This procedure was not applied for adiabatic corrections. The second kind of errors cannot be corrected, as it results from the approximate character 
of the electronic wave function of the interacting system. The accuracy of this function depends on the basis set, so we had to choose the appropriate basis for further calculations. Our decision was based on comparing the results for positronium hydride, for which accurate adiabatic potential energy curves were available from the earlier work. The values to be reproduced were positronic state energy and expectation value of $\left\langle\delta_{\mathrm{ep}}\right\rangle$ obtained with ECG functions (see Table I), for $q_{\mathrm{p}}=0.49$, which was found to be optimal for this system [32].

TABLE I

Basis set dependence of positronic state energies and electron-positron coalescences for PsH.

\begin{tabular}{c|c|c}
\hline \hline Basis & $E_{\mathrm{p}}[$ hartree] & $\left\langle\delta_{\mathrm{ep}}\right\rangle$ \\
\hline ECG & -0.02628 & 0.0431 \\
\hline $6-311 \mathrm{G}^{* *}$ & -0.02185 & 0.0382 \\
$6-311++\mathrm{G}^{* *}$ & -0.01973 & 0.0377 \\
$6-311++\mathrm{G}(2 \mathrm{~d}, 2 \mathrm{p})$ & -0.02231 & 0.0385 \\
ANO & -0.02316 & 0.0405
\end{tabular}

We started from the $6-311 \mathrm{G}^{* *}$ basis, containing a single shell of polarization functions at each atom. Adding diffuse functions $\left(6-311++\mathrm{G}^{* *}\right.$ basis $)$ resulted in deterioration of the results. This effect means that the energies of isolated hydrogen and positronium atoms (which do not profit from polarization functions at all, as they are one-electron systems) were lowered to a larger extent than that of the interacting system. Replacing one shell of polarization functions by two such shells in the $6-311++\mathrm{G}(2 \mathrm{~d}, 2 \mathrm{p})$ basis improved the potential, decreasing the difference between the reference value of $E_{\mathrm{p}}$ and the one computed with the CI method to less than 4 millihartrees. Going to a larger basis set of atomic natural orbitals (ANO, constructed by Roos et al. [33]) augmented by diffuse functions, resulted in further improvement of $E_{\mathrm{p}}$ by less than 1 millihartree, at the cost of significant increase in computation times. We planned to use also the augmented correlation consistent basis sets proposed by Dunning [34], but they are not available for lithium, beryllium and magnesium atoms. We decided to use the $6-311++\mathrm{G}(2 \mathrm{~d}, 2 \mathrm{p})$ basis [35], in order to scan a range of values of $q_{\mathrm{p}}$ and find its optimal value. Then, the ANO basis was used to compute single potential energy curve for this value of $q_{\mathrm{p}}$. It is important to notice that none of the considered basis sets overestimated the dissociation energy.

\section{Numerical results}

\subsection{Lithium positride, LiPs}

The structure of lithium positride is similar to that of positronium hydride. Its dissociation energy (to $\mathrm{Li}$ and Ps atoms) has been calculated with high accuracy, in the basis of explicitly correlated Gaussian functions with fixed electronic 


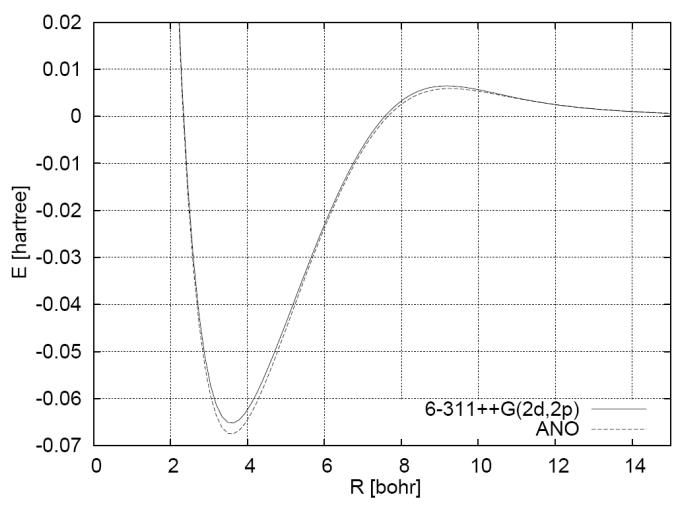

Fig. 1. Potential energy curves for positronic motion in lithium positride, $q_{\mathrm{p}}=0.49$.

core, and amounts to 0.012341 hartree [3]. Our potential energy curves were qualitatively similar to those computed for PsH. Examples for $q_{\mathrm{p}}=0.49$ are plotted in Fig. 1. However, the minimum is more shallow than in PsH and neither the plotted potentials nor those computed for different values of $q_{\mathrm{p}}$ support a bound state. This apparent failure of adiabatic approximation to provide at least a qualitatively correct description of a bound state puts in question the usefulness of our method in studies of positronium compounds.

\subsection{Positronic beryllium, $e^{+}$Be}

In our previous work we obtained quite accurate energy and annihilation rate for the model positronic atom with $Z=0.9$, of which dissociation energy was even smaller than that of $\mathrm{e}^{+} \mathrm{Be}$. After that, we expected that results of comparable quality could be obtained also for real systems having similar structure, i.e. a weakly bound positron, whose binding does not cause significant changes of the electronic structure. The potentials for the positronic motion supported bound states, but even the dissociation energy computed for optimal $q_{\mathrm{p}}=0.57$, with the basis of atomic natural orbitals, was underestimated by the factor of more

TABLE II

Positronic beryllium - comparison of the results obtained within our adiabatic formalism with other methods.

\begin{tabular}{l|c|c|c}
\hline \hline \multicolumn{1}{c|}{ Method } & $D_{0}[$ hartree $]$ & $\left\langle r_{\mathrm{p}}\right\rangle[\mathrm{bohr}]$ & $\left\langle\delta_{\mathrm{ep}}\right\rangle\left[\mathrm{bohr}^{-3}\right]$ \\
\hline ECG, fixed core $^{a}$ & 0.003180 & 10.048 & 0.00850 \\
CI, $l \leq 12^{a}$ & 0.002840 & 10.48 & 0.00562 \\
CI, extrapolated $^{a}$ & 0.003169 & 10.10 & 0.00819 \\
\hline$q_{\mathrm{p}}=0.57$, ANO & 0.000504 & 20.13 & 0.00345 \\
$q_{\mathrm{p}}=0.57,6-311++\mathrm{G}(2 \mathrm{~d}, 2 \mathrm{p})$ & 0.000340 & 23.67 & 0.00263 \\
\hline
\end{tabular}

${ }^{a}$ Ref. [18] 


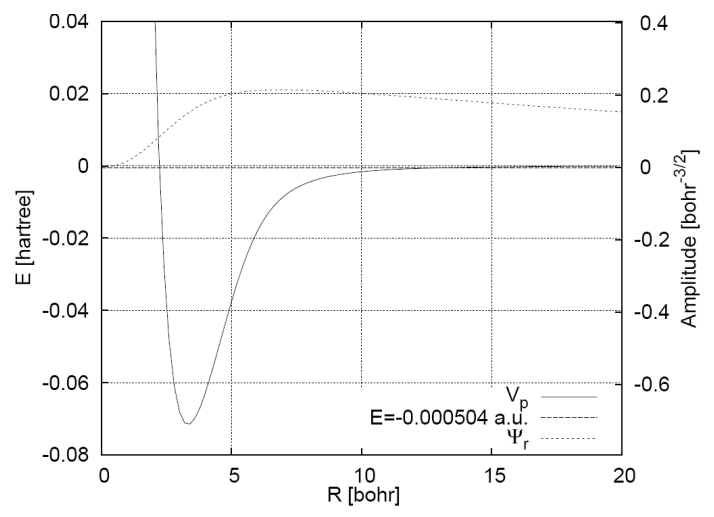

Fig. 2. Potential energy curve and positronic wave function of positronic beryllium for $q_{\mathrm{p}}=0.57$, ANO basis.

than 6 (Table II). The positronic wave function is very diffuse (see Fig. 2) and the average positron-nucleus distance is about twice larger than that obtained in reference calculations [18]. Consequently, the values of $\left\langle\delta_{\mathrm{ep}}\right\rangle$ are underestimated, as the positron described with such a diffuse function could not penetrate the electronic cloud. The difference between adiabatic dissociation energies, obtained with $6-311++\mathrm{G}(2 \mathrm{~d}, 2 \mathrm{p})$ and ANO basis sets amounts to $164 \mu$ hartrees only, so probably $D_{0}$ would remain significantly smaller than 1 millihartree even in the limit of infinite basis.

\subsection{Positronic magnesium, $e^{+} M g$}

The magnesium atom does bind the positron more tightly than beryllium. Our results and the most accurate values of dissociation energies of positronic magnesium and its electron-positron coalescences, available in the literature, have been collected in Table III. All potential energy curves computed by us supported bound states and the dependence of $E_{\mathrm{p}}$ on $q_{\mathrm{p}}$ for the $6-311++\mathrm{G}(2 \mathrm{~d}, 2 \mathrm{p})$ basis is

TABLE III

Positronic magnesium - comparison of the results obtained within our adiabatic formalism with other methods.

\begin{tabular}{l|c|c|c}
\hline \hline \multicolumn{1}{c}{ Method } & $D_{0}[$ hartree $]$ & $\left\langle r_{\mathrm{p}}\right\rangle[\mathrm{bohr}]$ & $\left\langle\delta_{\mathrm{ep}}\right\rangle\left[\mathrm{bohr}^{-3}\right]$ \\
\hline ECG, fixed core $^{a}$ & 0.016930 & 6.923 & 0.0203 \\
$\mathrm{CI}, l \leq 12^{a}$ & 0.015658 & 7.019 & 0.0128 \\
CI, extrapolated $^{a}$ & 0.017040 & 6.937 & 0.0198 \\
DMC $^{b}$ & $0.0168(14)$ & & \\
\hline$q_{\mathrm{p}}=0.573,6-311++\mathrm{G}(2 \mathrm{~d}, 2 \mathrm{p})$ & 0.009023 & 8.069 & 0.01649 \\
$q_{\mathrm{p}}=0.573$, ANO & 0.009984 & 7.867 & 0.01837 \\
\hline
\end{tabular}

${ }^{a}$ Ref. [18]; ${ }^{b}$ Ref. [12] 


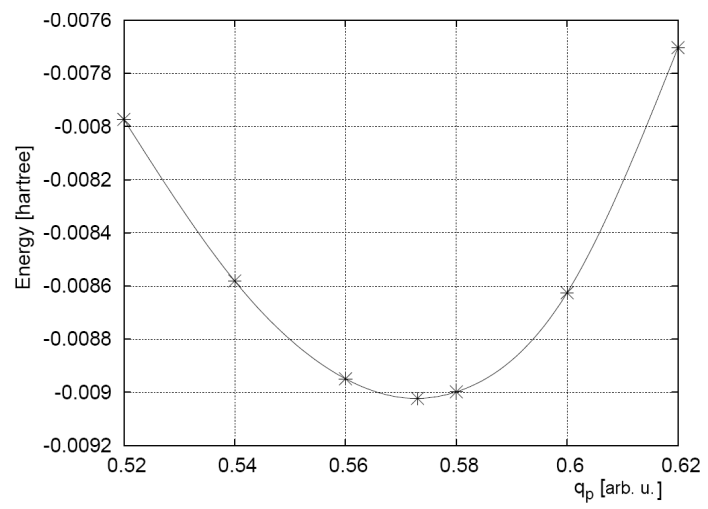

Fig. 3. Positronic state energy in $\mathrm{e}^{+} \mathrm{Mg}$ as function of effective positronic charge $q_{\mathrm{p}}$ for $6-311++\mathrm{G}(2 \mathrm{~d}, 2 \mathrm{p})$ basis.

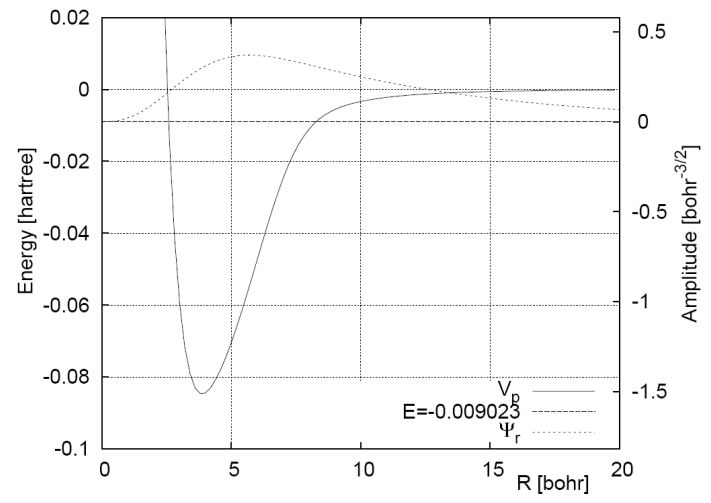

Fig. 4. Potential energy curve and positronic wave function of positronic magnesium for $q_{\mathrm{p}}=0.573,6-311++\mathrm{G}(2 \mathrm{~d}, 2 \mathrm{p})$ basis.

plotted in Fig. 3. We have to admit again that our method does not compare very well with accurate calculations, either variational (ECG and CI) or Monte Carlo simulations - the dissociation energy is underestimated by 7-8 millihartrees, so its absolute error is even twice larger than for positronic beryllium. However, because of the larger value of $D_{0}$, its relative error is close to only $40 \%$. The positronic wave function is much better localized in the minimum of the potential (Fig. 4) and reasonable values of the average positron-nucleus distance and electron-positron coalescence have been obtained for optimal $q_{\mathrm{p}}=0.573$.

\section{Discussion}

The results we obtained for lithium positride, positronic beryllium, and positronic magnesium, clearly indicate that the domain of applicability of our adiabatic method for the description of positronic bound states is narrower than 
we expected. For positronium compounds, like LiPs, this method may even appear to be unable to yield a bound state wave function. Serious underestimation of the dissociation energy of positronic beryllium means that the short-range correlations between electronic and positronic motions are more important for positron binding than polarization of the electronic wave function by the positron. These correlations appear to be nonadiabatic by their nature - the electrons follow the positron, but also the positron should follow the electrons. Only the calculation carried out for $\mathrm{e}^{+} \mathrm{Mg}$ atom may be regarded as partially successful, especially because of good value of $\left\langle\delta_{\mathrm{ep}}\right\rangle$. This success could be attributed to the dipole polarizability of magnesium, which is about twice larger than that of beryllium [36] and makes the effect of long-range polarization of the electron distribution relatively more important.

A practical remedy for the deficiencies of our adiabatic method could be possibly the inclusion of a heuristic nonadiabatic correction in the form of modified positron mass in Eq. (5). Such a modification, in which the averaged electron population on the positron is added to the positron mass, has been proposed recently by Mohallem et al. [37]. The price to be paid is the loss of variational bounds on the leptonic energy. Such an approach could be studied in a future work.

Finally, it should be asked why the adiabatic method worked well for model one-electron positronic atoms with fractional $Z$ in the range 0.7-0.9 [32]. The answer seems to be simple, but it has been overlooked by us, while working on the earlier article. In those atoms, static potentials were present, resulting from unbalanced total charges and supporting the positron binding even without taking the dynamic correlation into account. This oversight resulted in non-satisfied expectations related to atoms with weakly bound positron. On the other hand, similar static potentials (dipole, quadrupole) occur for many molecules, for which the positron binding still remains in the area of presumptions and could be possibly studied with our method.

\section{Acknowledgments}

This work was supported by Wrocław University of Technology. We are also grateful to the authors of freely available computer programs: PSI3 [38], which was used, after little modifications, for calculations of potential energy curves for positronic motion, and LEVEL [39] for solving the radial Schrödinger equation for positronic wave functions.

\section{References}

[1] G.G. Ryzhikh, J. Mitroy, Phys. Rev. Lett. 79, 4124 (1997).

[2] K. Strasburger, H. Chojnacki, J. Chem. Phys. 108, 3218 (1998).

[3] J. Mitroy, M.W.J. Bromley, G.G. Ryzhikh, J. Phys. B 35, R81 (2002).

[4] J. Mitroy, Phys. Rev. A 70, 024502 (2004).

[5] J. Mitroy, Phys. Rev. Lett. 94, 033402 (2005).

[6] J. Mitroy, Phys. Rev. A 72, 032503 (2005). 
[7] M.W.J. Bromley, J. Mitroy, K. Varga, Phys. Rev. A 75, 062505 (2007).

[8] J.-Y. Zhang, J. Mitroy, Phys. Rev. A 76, 014501 (2007).

[9] D. Bressanini, M. Mella, G. Morosi, J. Chem. Phys. 108, 4756 (1998).

[10] D. Bressanini, M. Mella, G. Morosi, J. Chem. Phys. 109, 1716 (1998).

[11] M. Mella, G. Morosi, D. Bressanini, J. Chem. Phys. 111, 108 (1999).

[12] M. Mella, M. Casalegno, G. Morosi, J. Chem. Phys. 117, 1450 (2002).

[13] M. Mella, D. Bressanini, G. Morosi, J. Chem. Phys. 114, 10579 (2001).

[14] G.F. Gribakin, J. Ludlow, J. Phys. B, At. Mol. Phys. 35, 339 (2002).

[15] M.W.J. Bromley, J. Mitroy, G.G. Ryzhikh, Nucl. Instrum. Methods Phys. Res. $B$ 171, 47 (2000).

[16] M.W.J. Bromley, J. Mitroy, Phys. Rev. A 65, 012505 (2002).

[17] M.W.J. Bromley, Ph.D. Thesis, Faculty of Science, Information Technology and Education, Northern Territory University, Australia 2002.

[18] M.W.J. Bromley, J. Mitroy, Phys. Rev. A 73, 032507 (2006).

[19] J. Mitroy, M.W.J. Bromley, Phys. Rev. A 73, 052712 (2006).

[20] M.W.J. Bromley, J. Mitroy, Phys. Rev. A 75, 042506 (2007).

[21] K. Strasburger, Chem. Phys. Lett. 253, 49 (1996).

[22] M. Tachikawa, R.J. Buenker, M. Kimura, J. Chem. Phys. 119, 5005 (2003).

[23] K. Strasburger, Struct. Chem. 15, 415 (2004).

[24] R.J. Buenker, H.P. Liebermann, V. Melnikov, M. Tachikawa, L. Pichl, J. Phys. Chem. A 109, 5956 (2005).

[25] H. Chojnacki, K. Strasburger, Mol. Phys. 104, 2273 (2006).

[26] H. Stachowiak, E. Boroński, Phys. Rev. B 71, 245107 (2005).

[27] F. Rolim, J.P. Braga, J.R. Mohallem, Chem. Phys. Lett. 332, 139 (2000).

[28] J.R. Mohallem, F. Rolim, C.P. Gonçalves, J. Phys. B 37, 1045 (2004).

[29] F. Rolim, T. Moreira, J.R. Mohallem, Brasilian J. Phys. 34, 1197 (2004).

[30] F. Rolim, J.R. Mohallem, Acta Phys. Pol. A 107, 661 (2005).

[31] S. Golden, I.R. Epstein, Phys. Rev. A 10, 761 (1974).

[32] K. Strasburger, M. Wołcyrz, Mol. Phys. 105, 441 (2007).

[33] P.O. Widmark, P.A. Malmqvist, B. Roos, Theor. Chim. Acta 77, 291 (1990).

[34] T.H. Dunning, Jr., J. Chem. Phys. 90, 1007 (1989).

[35] M.J. Frisch, J.A. Pople, J. Chem. Phys. 80, 3265 (1984).

[36] V. Magnasco, M. Amelio, J. Chem. Phys. 69, 4706 (1978).

[37] D. Assafraõ, H.R.J. Walters, J.R. Mohallem, Nucl. Instrum. Methods Phys. Res. $B$ 266, 491 (2008).

[38] PSI 3.2 by T.D. Crawford, C.D. Sherrill, E.F. Valeev, J.T. Fermann, R.A. King, M.L. Leininger, S.T. Brown, C.L. Janssen, E.T. Seidl, J.P. Kenny, W.D. Allen, http://www.psicode.org.

[39] LEVEL 8.0 by Robert J. Le Roy, Dept. of Chemistry, Univ. of Waterloo, Waterloo, Ontario, Canada. 